IPMU-08-0025

\title{
Solving Cosmological Problems of Supersymmetric Axion Models in Inflationary Universe
}

\author{
Masahiro Kawasaki ${ }^{a b}$ and Kazunori Nakayama ${ }^{a}$ \\ a Institute for Cosmic Ray Research, \\ University of Tokyo, Chiba 277-8582, Japan \\ ${ }^{b}$ Institute for the Physics and Mathematics of the Universe, \\ University of Tokyo, Chiba 277-8582, Japan
}

(Dated: October 27, 2018)

\begin{abstract}
We revisit inflationary cosmology of axion models in the light of recent developments on the inflaton decay in supergravity. We find that all the cosmological difficulties, including gravitino, axino overproduction and axionic isocurvature fluctuation, can be avoided if the saxion field has large initial amplitude during inflation and decays before big-bang nucleosynthesis.
\end{abstract}




\section{INTRODUCTION}

Although the standard model of the particle physics has achieved great successes, it still has some theoretical problems. One is the gauge hierarchy problem, and another is the strong CP problem. On the otherhand, cosmological problems such as dark matter and baryon asymmetry of the universe cannot be explained within the framework of the standard model. These problems indicate that there is an underlying physics beyond the standard model.

Supersymmetry (SUSY) is one of the best motivated candidates as physics beyond the standard model to solve the gauge hierarchy problem. On the other hand, Peccei-Quinn (PQ) mechanism is a simple promising solution to the strong $\mathrm{CP}$ problem [1]. As a consequence of the breaking of PQ symmetry, the existence of a pseudo-Nambu-Goldstone boson, axion, is predicted [2]. Thus we are eager to study the axion models within the framework of SUSY. However, cosmology of SUSY axion models is highly non-trivial. It is well known that gravitino, which is the fermionic superpartner of the graviton and has a long lifetime, is produced during reheating after inflation and its decay gives serious effect to thermal histry of the universe (this is called gravitino problem). In addition to the gravitino, there appear other long-lived particles called saxion, the scalar partner of the axion, and axino, the fermionic superpartner of the axion [3]. Both of them have a potential to cause cosmological disaster, which may be even more problematic than the usual gravitino problem [4, 5, 6, 7, 8, 9].

Furthermore, recently it is pointed out that gravitinos are also produced non-thermally from the inflaton decay [10, 11, 12, 13, 14, 15]. Inclusion of such contributions makes the gravitino problem much worse so that some inflation models may be excluded depending on the mass of the gravitino.

This is not the end of the story. The axion field induces an isocurvature fluctuation [16], whose amplitude is proportional to the Hubble scale during inflation. Recent cosmological observations are consistent with pure adiabatic one as the initial density fluctuation of

the universe [17], and hence the fraction of the isocurvature component to the density perturbation is constrained. This excludes the high-scale inflation models such as chaotic inflation or many hybrid inflation models.

Thus SUSY axion models seem to suffer from various cosmological problems, which ex- 
clude many inflation models. However, in this paper we point out that a resolution to all the above difficulties is already built in SUSY axion models themselves. The saxion is naturally expected to have a large initial amplitude of order of the reduced Planck scale $M_{P}$ during inflation, and starts coherent oscillation after inflation. Soon after reheating due to the inflaton decay, the saxion dominates the universe since the energy density of the saxion is almost comparable to the total energy density during the inflaton-dominated era. Finally, the saxion decays at later epoch and reheats the universe again releasing huge entropy. A remarkable feature is that huge entropy produced by the saxion decay dilutes the possibly harmful gravitinos and axinos. This provides a solution to the cosmological gravitino and axino problems. Moreover, the amplitude of the axionic isocurvature fluctuation is also significantly reduced for a large initial amplitude of the saxion, which makes high-scale inflation models such as chaotic inflation compatible with cosmological constraints.

This paper is organized as follows. In Sec II we overview cosmological problems with the gravitino, axino and axion. In Sec III, we show that entropy-production by the saxion decay overcomes these cosmological difficulties. We conclude in SecIV.

\section{COSMOLOGICAL PROBLEMS}

In this section we briefly summarize cosmological constraints on the abundances of longlived particles, the gravitino, axino and axion, which appear in SUSY axion models.

\section{A. Gravitino}

Gravitinos are produced in the early universe through scatterings of particles in thermal bath. For an unstable gravitino, its decay may significantly affect Big-Bang nucleosynthesis $(\mathrm{BBN})[19,20]$. If the gravitino is stable, it may have too large contribution to the present

matter density of the universe [21]. Thus the gravitino abundance is constrained for nearly all mass range. The abundance is calculated as [20, 22]

$$
\begin{array}{rl}
Y_{3 / 2}^{(\mathrm{TP})} \simeq 1 & 1.9 \times 10^{-12}\left(1+\frac{m_{\tilde{g}}^{2}}{3 m_{3 / 2}^{2}}\right)\left(\frac{T_{R}}{10^{10} \mathrm{GeV}}\right) \\
& \times\left[1+0.045 \ln \left(\frac{T_{R}}{10^{10} \mathrm{GeV}}\right)\right]\left[1-0.028 \ln \left(\frac{T_{R}}{10^{10} \mathrm{GeV}}\right)\right],
\end{array}
$$


where $m_{\tilde{g}}$ is the gluino mass and $T_{R}$ is the reheating temperature of the universe defined as $T_{R}=\left(10 / \pi^{2} g_{*}\right)^{1 / 4} \sqrt{\Gamma_{\text {total }} M_{P}}$.

Recently, it was pointed out that gravitinos are also produced directly from the decay of the inflaton through supergravity effects $[10,11,12,13]$. Taking account of the mixing of the inflaton with SUSY breaking field, the decay rate of the inflaton into gravitino pair is written as

$$
\Gamma\left(\phi \rightarrow \psi_{3 / 2} \psi_{3 / 2}\right) \simeq \frac{\left|\mathcal{G}_{\phi}\right|^{2}}{288 \pi} \frac{m_{\phi}^{5}}{m_{3 / 2}^{2} M_{P}^{2}},
$$

where $m_{\phi}$ denotes the inflaton mass and $\mathcal{G}_{\phi}$ is the effective coupling to the gravitino given in Ref. [12]. In this paper we assume a dynamical SUSY breaking scenario with dynamical scale $\Lambda$. If $m_{\phi} \ll m_{Z}$ where $m_{Z}$ denotes the mass of the SUSY breaking field (hereafter for simplicity we assume $m_{Z} \sim \Lambda$ ), the direct production process cannot be suppressed. In this case, the effective coupling is given by $\left|\mathcal{G}_{\phi}\right| \sim 3\left(\langle\phi\rangle / M_{P}\right)\left(m_{3 / 2} / m_{\phi}\right)$ and the decay rate is estimated as [12, 15]

$$
\Gamma\left(\phi \rightarrow \psi_{3 / 2} \psi_{3 / 2}\right) \simeq \frac{1}{32 \pi}\left(\frac{\langle\phi\rangle}{M_{P}}\right)^{2} \frac{m_{\phi}^{3}}{M_{P}^{2}} .
$$

This gives the gravitino abundance as

$$
\begin{aligned}
Y_{3 / 2}^{(\mathrm{NTP})} & \simeq 2 \frac{\Gamma\left(\phi \rightarrow \psi_{3 / 2} \psi_{3 / 2}\right)}{\Gamma_{\text {total }}} \frac{3 T_{R}}{4 m_{\phi}} \\
& \simeq 7 \times 10^{-11}\left(\frac{\langle\phi\rangle}{10^{15} \mathrm{GeV}}\right)^{2}\left(\frac{m_{\phi}}{10^{12} \mathrm{GeV}}\right)^{2}\left(\frac{T_{R}}{10^{6} \mathrm{GeV}}\right)^{-1} .
\end{aligned}
$$

If $m_{\phi}>\Lambda$, the direct decay of the inflaton into gravitino pair can be suppressed if there are no couplings such as $\delta K \sim|\phi|^{2} z z$ in the Kähler potential. Instead, the inflaton decays into hidden gauge sector through the anomaly effects, and each hidden hadron eventually produces at least one gravitino [14]. The partial decay rate of the inflaton into hidden gauge sector is given by

$$
\Gamma_{\text {anomaly }} \simeq \frac{N_{h} \alpha_{h}^{2}}{256 \pi^{3}}\left(\mathcal{T}_{G}^{h}-\mathcal{T}_{R}^{h}\right)^{2}\left(\frac{\langle\phi\rangle}{M_{P}}\right)^{2} \frac{m_{\phi}^{3}}{M_{P}^{2}},
$$

where $N_{h}$ is the number of generators and $\alpha_{h}$ is the gauge coupling constant of the hidden gauge group. $\mathcal{T}_{G}^{h}$ and $\mathcal{T}_{R}^{h}$ are the Dynkin index of the adjoint representation and matter fields in the representation of the dimension $d_{R}$, respectively. Then the abundance of nonthermally produced gravitinos is given by

$$
\begin{aligned}
Y_{3 / 2}^{(\mathrm{NTP})} & \simeq 2 N_{3 / 2} \frac{\Gamma_{\text {anomaly }}}{\Gamma_{\text {total }}} \frac{3 T_{R}}{4 m_{\phi}} \\
& \simeq 9 \times 10^{-13} \epsilon\left(\frac{\langle\phi\rangle}{10^{15} \mathrm{GeV}}\right)^{2}\left(\frac{m_{\phi}}{10^{12} \mathrm{GeV}}\right)^{2}\left(\frac{T_{R}}{10^{6} \mathrm{GeV}}\right)^{-1},
\end{aligned}
$$


where $\epsilon$ is $O(1)$ constant given by $\epsilon=N_{3 / 2} N_{h} \alpha_{h}^{2}\left(\mathcal{T}_{G}^{h}-\mathcal{T}_{R}^{h}\right)^{2}$ (here $N_{3 / 2}$ denotes the averaged number of produced gravitinos per hidden hadron jet).

If the gravitino is unstable, photons or hadrons produced through the decay of the gravitino may affect light element abundances synthesized through BBN. On the other hand, if the gravitino is stable, it contributes to the present dark matter density. Both set the upper bound on the gravitino abundance and reheating temperature of the universe. Moreover, we can see that $Y_{3 / 2}^{(\mathrm{TP})}$ is proportional to $T_{R}$, while $Y_{3 / 2}^{(\mathrm{NTP})}$ is proportional to $T_{R}^{-1}$. Thus lowering $T_{R}$ does not ameliorate the situation. Also it was found that the reheating temperature of the universe is bounded from below, due to the spontaneous decay processes of the inflaton through top Yukawa coupling [23], as

$$
T_{R} \gtrsim 3 \mathrm{TeV}\left|y_{t}\right|\left(\frac{228.75}{g_{*}\left(T_{R}\right)}\right)^{1 / 4}\left(\frac{m_{\varphi}}{10^{12} \mathrm{GeV}}\right)^{3 / 2}\left(\frac{\langle\phi\rangle}{10^{15} \mathrm{GeV}}\right)
$$

where $y_{t}$ is the top Yukawa coupling. Including non-thermally produced contribution, many inflation models are severely constrained. For example, if the gravitino mass is $\sim 1 \mathrm{TeV}$ and its hadronic branching ratio is $O(1)$, typical inflation models such as new, hybrid and chaotic inflation models are excluded [15].

\section{B. Axino}

In SUSY axion model, the axion forms a supemultiplet which includes scalar partner of the axion called saxion, and fermionic superpartner of the axion called axino. Both of them have long lifetime and may significantly affect cosmology [4, 5, 6, 7, 8, 9]. Similar to the gravitino, axinos are also produced through scatterings of the particles in thermal bath. The resulting abundance is calculated as [5]

$$
Y_{\tilde{a}} \simeq 2.0 \times 10^{-7} g_{s}^{6}\left(\frac{F_{a}}{10^{12} \mathrm{GeV}}\right)^{-2}\left(\frac{T_{R}}{10^{6} \mathrm{GeV}}\right),
$$

where $g_{s}$ is the QCD gauge coupling constant. This expression is valid for $T_{R} \gtrsim 10 \mathrm{TeV}$, and the abundance is suppressed for $T_{R} \lesssim 1 \mathrm{TeV}$ because SUSY particles are not produced efficiently. Although the axino mass is model dependent, it may have the mass of order of the gravitino mass [24]. Here we assume that the axino mass is of the order of the gravitino mass. 
From (8), one can see that very low reheating temperature is needed in order to avoid the overproduction of the axino if it is the LSP. This sets an upper bound on $T_{R}$ as $T_{R} \lesssim 1 \mathrm{TeV}$ for $m_{\tilde{a}} \gtrsim 1 \mathrm{GeV}$, and $T_{R} \lesssim 1 \mathrm{TeV}\left(1 \mathrm{GeV} / m_{\tilde{a}}\right)$ for $m_{\tilde{a}} \lesssim 1 \mathrm{GeV}$ when $F_{a}=10^{11} \mathrm{GeV}$.

\section{Axion}

The axion is a pseudo-Nambu-Goldstone boson associated with spontaneous breaking of the PQ symmetry. The axion is practically massless for $T \gtrsim 1 \mathrm{GeV}$ due to the finitetemperature effect, and begins to oscillate after it becomes massive for $T \lesssim 1 \mathrm{GeV}$. The abundance of axion in the form of coherent oscillation is estimated as [25]

$$
\Omega_{a} h^{2} \sim 0.2\left(\frac{F_{a} \theta_{i}^{1.7}}{10^{12} \mathrm{GeV}}\right)^{1.18}
$$

where $\theta_{i}$ denotes the initial misalignment angle of the axion, which is naturally expected to be $O(1)$ without fine-tuning. Thus the upper bound on the PQ scale is $F_{a} \lesssim 10^{12} \mathrm{GeV}$, although late-time entropy production can relax this upper bound [26]. On the other hand, astrophysical arguments require that $F_{a}$ should be larger than $\sim 10^{10} \mathrm{GeV}[27]$.

If the PQ symmetry is broken during or before the inflation, the quantum fluctuation of the axion induces isocurvature fluctuation with magnitude $\sim H_{I} /\left(\pi s_{i}\right)$ [16], where $s_{i}$ denotes the field value of the saxion during inflation. Recent cosmological observations indicate that the ratio of the magnitude of isocurvature perturbation to adiabatic one should be less than about $0.3[17,18]$. This leads to the constraint on the Hubble scale during inflation,

$$
H_{I} \lesssim 2 \times 10^{7} \mathrm{GeV} \theta_{i}^{-1}\left(\frac{\Omega_{m} h^{2}}{0.13}\right)\left(\frac{s_{i}}{F_{a}}\right)\left(\frac{F_{a}}{10^{12} \mathrm{GeV}}\right)^{-0.18} .
$$

Thus, high-scale inflation models are excluded unless $s_{i}$ is as large as the Planck scale. As a result, low-scale inflation models such as a new inflation model were considered to be suitable for the axion cosmology. Note that if $H_{I} \gtrsim F_{a}$, the PQ symmetry may be restored during inflation and hence isocurvature constraint does not apply, although there may be problematic domain wall formation for generic axion models.

In Fig. 1 the axion isocurvature constraint is shown on the $m_{\phi}-\langle\phi\rangle$ plane with $s_{i}=F_{a}=$ $10^{12} \mathrm{GeV} .{ }^{1}$ Also we show the prediction of typical inflation models in supergravity, new

\footnotetext{
${ }^{1}$ Here we have estimated the inflation energy scale by $H_{I} \sim m_{\phi}\langle\phi\rangle / \sqrt{3} M_{P}$. For inflation models with the
} 


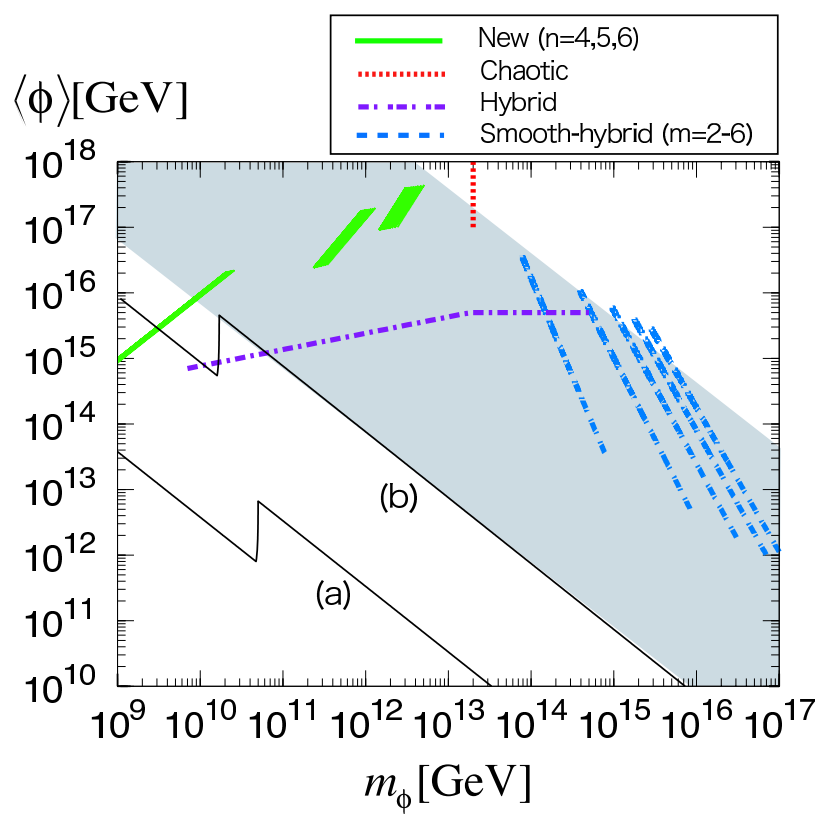

FIG. 1: Axion isocurvature constraint on the $m_{\phi^{-}}\langle\phi\rangle$ plane with $s_{i}=F_{a}=10^{12} \mathrm{GeV}$. The shaded region is excluded from the isocurvature perturbation constraint. Green solid lines represent new inflation models with $n=4,5,6$ from left to right, red dotted line represents chaotic inflation model, purple dot-dashed line represents hybrid-inflation model and blue dashed lines represent smoothhybrid inflation models for $m=2-6$ from left to right (for definitions of $n$ and $m$ see footnote). Region above thin solid black lines are excuded from gravitino overproduction in inflaton decay for (a) $m_{3 / 2}=1 \mathrm{TeV}$ and (b) $m_{3 / 2}=100 \mathrm{GeV}$, with $T_{R}=1 \mathrm{TeV}$.

[28, 29], hybrid [30, 31], smooth-hybrid [32], and chaotic inflation [33] models. ${ }^{2}$ Furthermore, non-thermally produced gravitinos restrict the parameter region. Regions above thin solid black lines are excluded from gravitino overproduction, for (a) $m_{3 / 2}=1 \mathrm{TeV}$ and (b) $m_{3 / 2}=100 \mathrm{GeV}$. Here we set $T_{R}=1 \mathrm{TeV}$, since axino thermal production sets the upper bound on the reheating temperature as $T_{R} \lesssim 1 \mathrm{TeV}$. Saxions are not harmful for cosmology for these parameter sets. We can see that most inflation models are excluded. However, if one allows a large field value of the saxion during inflation, the effective PQ scale during inflation

potential like $V \sim\left(v^{2}-g \phi^{n}\right)^{2}$, as is the case for new, hybrid and smooth-hybrid inflation models, this estimation is correct except for the numerical factor $n$. For the chaotic inflation model, this evaluation cannot be applied and the correct estimate is $H_{I} \sim 10^{14} \mathrm{GeV}$.

2 We assume the following superpotentials for new and smooth hybrid inflation models : $W=\psi\left(v^{2}-g \phi^{n}\right)$ for new inflation and $W=\psi\left[v^{2}-(\bar{\phi} \phi)^{m} / M^{2 m-2}\right]$ for smooth hybrid new inflation model. 
can be practically much larger than $F_{a}$, which suppress the magnitude of the isocurvature perturbation [34], as we will see in the next section.

\section{SAVING INFLATION MODELS}

All the above cosmological difficulties are avoided by taking account of the dynamics of the saxion. In the above arguments, we have assumed that there was no entropy production after reheating by the inflaton. However, in SUSY axion model, the saxion can have the large initial amplitude of order of the Planck scale. For example, let us consider the case where the saxion has negative Hubble mass term. Since the saxion $s$ corresponds to a flat direction in the scalar potential, the saxion field rolls away due to the negative Hubble mass term during inflation until the field value becomes the Planck scale where the potential becomes steep as $\sim \exp \left(s^{2} / M_{P}^{2}\right)$. Such saxion condensate provides a source of late-time entropy production [35] and dilutes the harmful gravitino and axino. Furthermore, large initial amplitude during inflation suppresses the axionic isocurvature fluctuation. Therefore, there arises a possibility that many inflation models are free from cosmological difficulties which we encountered in the previous section.

\section{A. Saxion with large initial amplitude}

The saxion corresponds to a flat direction along which the PQ scalars do not feel the potential. A flat direction is lifted by the SUSY breaking effect and hence the saxion mass $\left(m_{s}\right)$ is naturally expected to be of order of the gravitino mass.

The coherent saxion oscillation starts at $H \sim m_{s}$, with the initial amplitude $s_{i}$. If $s_{i} \sim F_{a}$ and the saxion is relatively light, its decay causes various cosmological difficulties, which severely restricts the saxion abundance [8, 9]. As a result, the upper bond on the reheating temperature becomes much more stringent than the case where the saxion and axino are absent for wide range of the saxion mass [9].

Here we consider another possibility. Thermal history of the universe can be significantly modified due to the saxion coherent oscillation with a large initial amplitude $s_{i} \sim M_{P}$. In this case the saxion dominates the universe immediately after the inflaton decays, and finally the universe is reheated again by the saxion decay. However, if the saxion main decay mode 
is into two axions $(s \rightarrow 2 a)$, the produced axions contribute to the extra relativistic degrees of freedom, which changes the Hubble expansion rate and the BBN prediction. Thus we need to investigate the saxion decay modes in order to ensure that the saxion decay does not produce too many axions. The decay rate into two axions is estimated as [24]

$$
\Gamma(s \rightarrow 2 a) \simeq \frac{f^{2}}{64 \pi} \frac{m_{s}^{3}}{F_{a}^{2}},
$$

where $f=\sum_{i} q_{i}^{3} v_{i}^{2} / F_{a}^{2}$ with the VEV of the $i$-th PQ scalar field $v_{i}$ and its PQ charge $q_{i}$. This often gives the dominant contribution to the total saxion decay rate if $f \sim 1$. In order to realize consistent cosmology, this must not be the main decay mode.

Another important decay mode is into gluons, arising from the coupling through the QCD anomaly effect. The decay rate is estimated as

$$
\Gamma_{s}(s \rightarrow g g) \simeq \frac{\alpha_{s}^{2}}{32 \pi^{3}} \frac{m_{s}^{3}}{F_{a}^{2}} .
$$

For the DFSZ axion model [36], the saxion can also decay into fermion-anti-fermion pair through the coupling arising from mixing of the PQ scalar with MSSM Higgs doublets. The decay rate is estimated as

$$
\Gamma\left(s \rightarrow d_{i} \bar{d}_{i}\right) \simeq \frac{3}{8 \pi}\left(\frac{2 x}{x+x^{-1}}\right)^{2} m_{s}\left(\frac{m_{d i}}{F_{a}}\right)^{2}\left(1-\frac{4 m_{d i}^{2}}{m_{s}^{2}}\right)^{3 / 2},
$$

for the decay into down-type quarks $d_{i}(i=1,2,3)$ where $x=\tan \beta=\left\langle H_{u}\right\rangle /\left\langle H_{d}\right\rangle$, and

$$
\Gamma\left(s \rightarrow u_{i} \bar{u}_{i}\right) \simeq \frac{3}{8 \pi}\left(\frac{2 x^{-1}}{x+x^{-1}}\right)^{2} m_{s}\left(\frac{m_{u i}}{F_{a}}\right)^{2}\left(1-\frac{4 m_{u i}^{2}}{m_{s}^{2}}\right)^{3 / 2} .
$$

for the decay into up-type quarks $u_{i}(i=1,2,3)$. The decay rate into Higgs boson pair is also comparable,

$$
\Gamma(s \rightarrow h h) \simeq \frac{1}{8 \pi} \frac{m_{s}^{3}}{F_{a}^{2}}\left(\frac{\mu}{m_{s}}\right)^{4}\left(1-\frac{4 m_{h}^{2}}{m_{s}^{2}}\right)^{1 / 2},
$$

where $\mu$-parameter is of order of the weak scale and set to $300 \mathrm{GeV}$ here. For simplicity we consider only the decay into the lightest Higgs bosons. Decays into other Higgs bosons also have comparable rate if kinematically allowed. Also we neglect decay into SUSY particle pair assuming that such decay modes are kinematically forbidden.

In order not to contradict with observations, the increase of the effective number of neutrino species $\Delta N_{\nu}$ should be smaller than about 1, which restricts the branching ratio into axions $B_{a}$ as

$$
\frac{B_{a}}{1-B_{a}} \lesssim \frac{7}{43}\left(\frac{g_{*}\left(T_{s}\right)}{10.75}\right)^{1 / 3} \Delta N_{\nu \text { (bound })}
$$




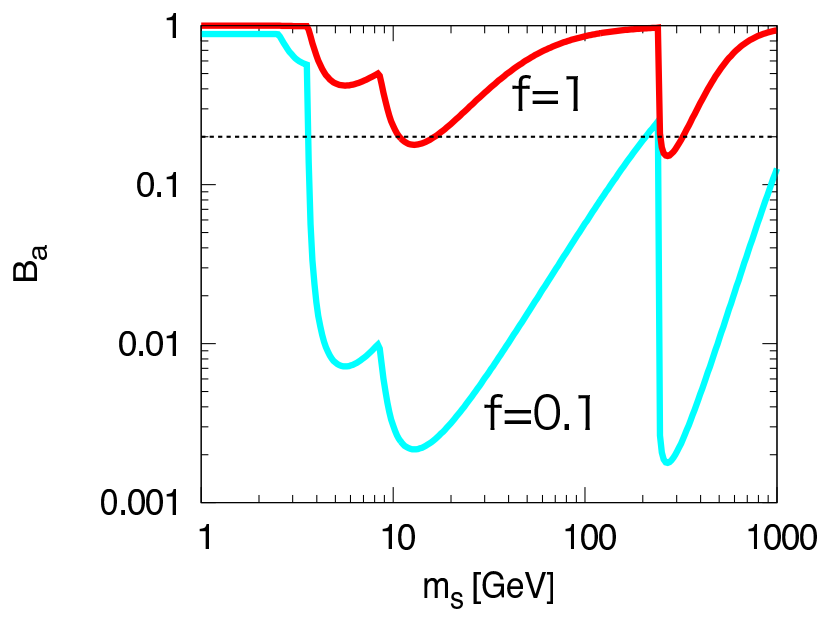

FIG. 2: Branching ratio into axions in DFSZ model for $f=1$ (upper) and $f=0.1$ (lower). Region above the thin dashed line is excluded.

where $\Delta N_{\nu \text { (bound) }} \sim 1$, and $T_{s}$ denotes the decay temperature of the saxion.

In Fig. 2, $B_{a}$ as a function of the saxion mass with $f=1$ and $f=0.1$ is shown. As a representative value, we show $B_{a}=0.2$ by the thin dashed line above which too many axions are produced by the saxion decay and the constraint (16) is not satisfied. It can be seen that for some mass ranges, the decay into axions is subdominant process. For that case the saxion is an ideal candidate of the source of late-time entropy production.

\section{B. Dilution of gravitinos and axinos}

We have shown that late-time entropy production by the saxion decay can can take place without producing too many axions. Now let us investigate the gravitino and axino abundances after the dilution by the saxion decay.

If we assume the main decay mode is $s \rightarrow b \bar{b}$, the typical decay temperature of the saxion is estimated as

$$
T_{s} \sim 250 \mathrm{MeV}\left(\frac{g_{*}\left(T_{s}\right)}{10}\right)^{-1 / 4}\left(\frac{m_{s}}{100 \mathrm{GeV}}\right)^{1 / 2}\left(\frac{F_{a}}{10^{11} \mathrm{GeV}}\right)^{-1},
$$

which is much lower than the typical reheating temperature after inflation. Thus, possibly harmful axinos and gravitinos, produced either thermally or non-thermally, are diluted by the saxion decay. After the dilution, thermally produced gravitinos during reheating pro-

cesses after inflation have only negligible abundance of order $Y_{3 / 2}^{(\mathrm{TP})} \lesssim 10^{-20}$ independently of 
$T_{R}$. On the other hand, the gravitino abundance produced from inflaton decay is estimated as

$$
\begin{aligned}
Y_{3 / 2}^{(\mathrm{NTP})} & =\frac{9 \Gamma\left(\phi \rightarrow \psi_{3 / 2} \psi_{3 / 2}\right)}{2 \Gamma_{\text {total }}} \frac{T_{s}}{m_{\phi}}\left(\frac{M_{P}}{s_{i}}\right)^{2} \gamma \\
& \sim 2.0 \times 10^{-12} \gamma\left(\frac{T_{s}}{1 \mathrm{GeV}}\right)\left(\frac{T_{R}}{10^{7} \mathrm{GeV}}\right)^{-2}\left(\frac{m_{\phi}}{10^{15} \mathrm{GeV}}\right)^{2}\left(\frac{\langle\phi\rangle}{10^{15} \mathrm{GeV}}\right)^{2}\left(\frac{M_{P}}{s_{i}}\right)^{2},
\end{aligned}
$$

for $m_{\phi}<\Lambda$, and

$$
\begin{aligned}
Y_{3 / 2}^{(\mathrm{NTP})} & =\frac{9 N_{3 / 2} \Gamma_{\text {anomaly }}}{2 \Gamma_{\text {total }}} \frac{T_{s}}{m_{\phi}}\left(\frac{M_{P}}{s_{i}}\right)^{2} \gamma \\
& \sim 2.6 \times 10^{-14} \epsilon \gamma\left(\frac{T_{s}}{1 \mathrm{GeV}}\right)\left(\frac{T_{R}}{10^{7} \mathrm{GeV}}\right)^{-2}\left(\frac{m_{\phi}}{10^{15} \mathrm{GeV}}\right)^{2}\left(\frac{\langle\phi\rangle}{10^{15} \mathrm{GeV}}\right)^{2}\left(\frac{M_{P}}{s_{i}}\right)^{2},
\end{aligned}
$$

for $m_{\phi}>\Lambda$ where $\gamma$ is defined as

$$
\gamma=\left\{\begin{array}{lll}
1 & \text { for } & T_{\mathrm{osc}}>T_{R} \\
T_{R} / T_{\mathrm{osc}} & \text { for } & T_{\mathrm{osc}}<T_{R}
\end{array},\right.
$$

with the temperature at which the saxion starts to oscillate $T_{\mathrm{osc}}$. Axinos are also diluted by the saxion decay and the resultant abundance is estimated $\mathrm{as}^{3}$

$$
Y_{\tilde{a}} \simeq 6.0 \times 10^{-13} g_{s}^{6}\left(\frac{F_{a}}{10^{12} \mathrm{GeV}}\right)^{-2}\left(\frac{T_{s}}{1 \mathrm{GeV}}\right)\left(\frac{M_{P}}{s_{i}}\right)^{2},
$$

In Figs. 3 and 4, the allowed and excluded region in $m_{\phi^{-}}\langle\phi\rangle$ plane are shown for the unstable gravitino with $m_{3 / 2}=1 \mathrm{TeV}$ and stable gravitino with $m_{3 / 2}=100 \mathrm{GeV}$. We put a constraint $Y_{3 / 2}<10^{-16}$ for the former case [37]. Note that axinos decay well before BBN for $m_{\tilde{a}} \gtrsim 1 \mathrm{TeV}$ and do not affect BBN. Instead, the LSP abundance emitted from the axino puts a constraint on the axino abundance, and this is also satisfied. It can be seen that all of the inflation models are allowed for the reheating temperature $T_{R} \gtrsim 10^{9}\left(10^{6}\right) \mathrm{GeV}$ for $m_{3 / 2}=1 \mathrm{TeV}$ $(100 \mathrm{GeV})$. Moreover, the constraint on the inflation scale (10) is relaxed for $s_{i} \sim M_{P}$, which enables even the chaotic inflation model to be consistent with observations. ${ }^{4}$

\footnotetext{
${ }^{3}$ Here we have assumed that the reheating due to the inflaton decay completes after the saxion oscillation. Otherwise, the axino abundance is suppressed by $s_{i}^{2} \sim M_{P}^{2}$, not $F_{a}^{2}$, and is safely neglected.

4 Again it should be noted that isocurvature constraint on the chaotic inflation model cannot be read from these figures. See the footnote in Sec II C
} 


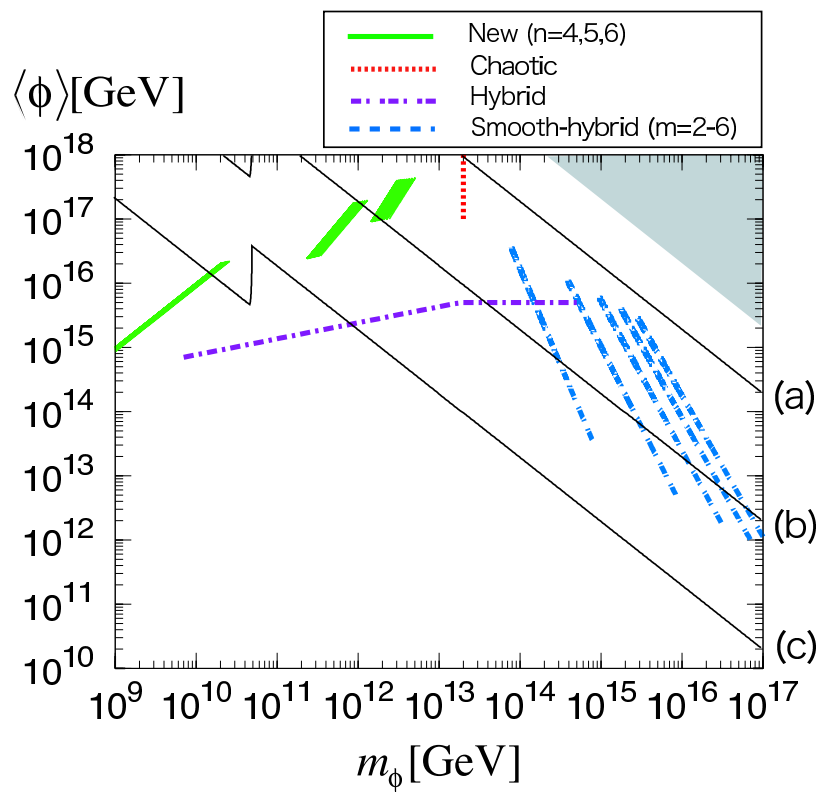

FIG. 3: Constraints on inflation models for $m_{3 / 2}=1 \mathrm{TeV}$ and $F_{a}=10^{12} \mathrm{GeV}$ with $s_{i}=M_{P}$. Region above the thin solid black lines is excluded for (a) $T_{R}=10^{9} \mathrm{GeV}$, (b) $T_{R}=10^{7} \mathrm{GeV}$ and (c) $T_{R}=10^{5} \mathrm{GeV}$. The shaded region is excluded from axionic isocurvature constraint.

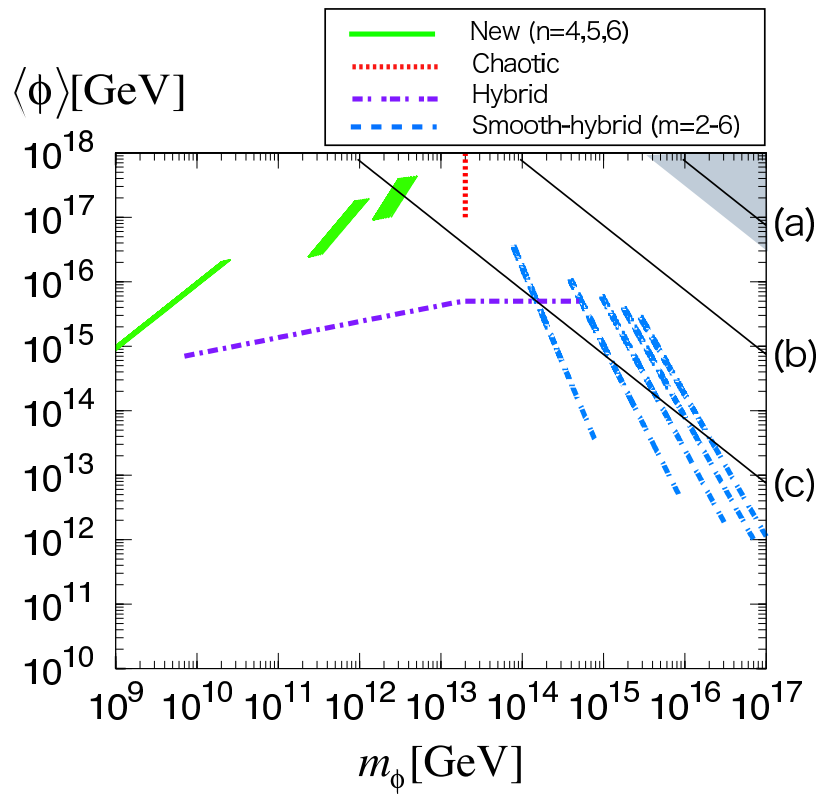

FIG. 4: Same as Fig. 3, except for $m_{3 / 2}=100 \mathrm{GeV}$ and $F_{a}=10^{11} \mathrm{GeV}$. 
Thus gravitinos are sufficiently diluted so that they do not cause cosmological difficulties. Interestingly, for the gravitino mass $m_{3 / 2} \sim 100 \mathrm{GeV}$, non-thermally produced gravitinos can have the desired abundance as dark matter of the universe. One issue to be addressed is the free-streaming length of the gravitino dark matter. It is calculated as

$$
\lambda_{\mathrm{FS}}=\int_{t_{i}}^{t_{\mathrm{eq}}} \frac{v(t)}{a(t)} d t \sim R_{\mathrm{eq}} u_{\mathrm{eq}} \ln \left[\frac{1}{u_{\mathrm{eq}}}+\sqrt{1+\frac{1}{u_{\mathrm{eq}}^{2}}}\right],
$$

where $R_{\text {eq }}$ is the comoving Hubble scale $(\sim 108 \mathrm{Mpc})$ and $u_{\mathrm{eq}}=v / \sqrt{1-v^{2}}$ with the velocity of the gravitino $v$, both are evaluated at the time of matter-radiation equality. $u_{\text {eq }}$ is estimated as

$$
u_{\text {eq }} \sim 8 \times 10^{-9}\left(\frac{m_{\phi}}{10^{15} \mathrm{GeV}}\right)\left(\frac{m_{3 / 2}}{1 \mathrm{TeV}}\right)^{-1}\left(\frac{T_{s}}{1 \mathrm{GeV}}\right)^{1 / 3}\left(\frac{T_{R}}{10^{8} \mathrm{GeV}}\right)^{-4 / 3} .
$$

Hence the free-streaming length is negligibly small and the gravitino dark matter acts as cold dark matter. The axion is diluted by the saxion decay because $T_{s} \lesssim 1 \mathrm{GeV}$ for $m_{s} \lesssim 100 \mathrm{GeV}$, but it may still have the comparable abundance to the present dark matter abundance.

For $m_{s} \gtrsim 1 \mathrm{TeV}$, there arises a possibility that the saxion decays into SUSY particles if the decay is kinematically allowed. According to Ref. [38], the decay rate of the saxion into gauginos are roughly the same as that into gauge bosons. Thus in general LSPs are overproduced by the saxion decay. However, for the LSP with rather large annihilation cross section, these non-thermally produced LSP abundance is reduced. The abundance is given by

$$
\frac{\rho_{\mathrm{LSP}}}{s} \simeq \min \left[B_{s} \frac{2 m_{\mathrm{LSP}}}{m_{s}} \frac{\rho_{s}}{s}, \sqrt{\frac{45}{8 \pi^{2} g_{*}\left(T_{s}\right)}} \frac{m_{\mathrm{LSP}}}{\langle\sigma v\rangle T_{s} M_{P}}\right],
$$

where $B_{s}$ denotes the branching ratio of the saxion into SUSY particles, and $\langle\sigma v\rangle$ denotes the thermally averaged annihilation cross section of the LSP. For the LSP with $\langle\sigma v\rangle \sim$ $10^{-7} \mathrm{GeV}^{-2}$, as is realized for the case of wino- or higgsino-like LSP with the mass of $O(100) \mathrm{GeV}$ [39], or bino-like LSP in the $S$-channel resonance region [40], the resultant LSP abundance can account for the present dark matter of the universe. Also there is contribution from the axion coherent oscillation to the dark matter abundance. Thus the dark matter may consist of a mixture of the axions and non-thermally produced LSPs. 


\section{Baryon asymmetry}

Note that baryon asymmetry is also diluted by the saxion decay. Here we show that Affleck-Dine mechanism [41] can create appropriate amount of baryon asymmetry. ${ }^{5}$ Let us denote the Affleck-Dine (AD) field, which is one of the flat directions in the scalar potential in the MSSM, as $\psi$. The flat direction is lifted by SUSY breaking effects and non-renormalizable terms in the superpotential $W_{\mathrm{NR}}=\psi^{n} / n M^{n-3}$ where $n(\geq 4)$ is an integer and $M$ denotes the cutoff scale. The resultant baryon asymmetry created by the coherent motion of the AD field is estimated as

$$
\frac{n_{B}}{s}=\frac{n_{B}}{\rho_{s}} \frac{\rho_{s}}{s} \simeq \frac{\delta_{\mathrm{CP}} m_{3 / 2}\left|\psi_{\mathrm{os}}\right|^{2}}{m_{\psi}^{2} s_{i}^{2}} \frac{3 T_{s}}{4}
$$

where $\delta_{\mathrm{CP}}$ denotes the effective $\mathrm{CP}$ angle, which is naturally expected to be $O(1)$, and $\psi_{\text {os }}$ is the field value at the onset of the oscillation of the $\mathrm{AD}$ field. If there exists a negative Hubble mass term for the AD field, the field value is given by $\left|\psi_{\text {os }}\right| \sim\left(m_{\psi} M^{n-3}\right)^{1 /(n-2)}$. For the specific case $n=6$, we obtain

$$
\frac{n_{B}}{s} \sim 2 \times 10^{-11} \delta_{\mathrm{CP}}\left(\frac{m_{\psi}}{1 \mathrm{TeV}}\right)^{-3 / 2}\left(\frac{m_{3 / 2}}{1 \mathrm{TeV}}\right)\left(\frac{T_{s}}{1 \mathrm{GeV}}\right)\left(\frac{M}{M_{P}}\right)^{3 / 2}\left(\frac{M_{P}}{s_{i}}\right)^{2}
$$

and hence AD mechanism works well. Although relatively large Q-balls are formed through the $\mathrm{AD}$ mechanism $\left(Q \sim 10^{20}\right)$ [43], their cosmological effects are safely neglected, since Q-balls decay before the saxion decays and LSPs emitted non-thermally by the Q-ball decay is diluted by the entropy-production from the saxion. ${ }^{6}$

\section{CONCLUSIONS}

We have shown that in SUSY axion models the saxion decay naturally dilutes the axino and gravitino abundances produced both thermally or non-thermally if the initial amplitude is of the order of $M_{P}$. The large initial amplitude of the saxion also saves high-scale inflation models such as chaotic inflation and hybrid inflation models from producing too much axionic

\footnotetext{
5 See e.g., Ref. [42] for more details in the case of late-time entropy production.

6 The Affleck-Dine mechanism described above has the baryonic isocurvature problem if the Hubble induced $A$-term is absent as expected for most inflation models [44]. In this case high scale infaltion models are disfavored. However, the problem is avoided for AD mechanism without superpotential [42].
} 
isocurvature fluctuation. Note that the preexisting baryon asymmetry is also diluted by the saxion. But the Affleck-Dine mechanism can create large baryon asymmetry which survives the dilution.

Finally we mention another possibility to dilute the gravitino and axino abundances. One may consider that thermal inflation [45, 46] can provide sufficient dilution. If the PQ scalar is trapped at the origin during inflation and remains there until later epoch due to the finite-temperature effect, the PQ scalar itself can cause a thermal inflation for some class of models [47]. Axionic isocurvature fluctuation does not arise since the PQ symmetry is restored during inflation. However, we must take care of topological defects formation after the onset of the PQ scalar oscillation, which may spoil the subsequent cosmological evolution scenario.

\section{Acknowledgment}

K.N. would like to thank the Japan Society for the Promotion of Science for financial support. This work was supported in part by the Grant-in-Aid for Scientific Research from the Ministry of Education, Science, Sports, and Culture of Japan, No. 18540254 and No 14102004 (M.K.). This work was also supported in part by JSPS-AF Japan-Finland Bilat-

eral Core Program (M.K.). This work was also supported by World Premier International Research Center InitiativeiWPI Initiative), MEXT, Japan.

[1] R. D. Peccei and H. R. Quinn, Phys. Rev. Lett. 38, 1440 (1977).

[2] For a review, see J. E. Kim, Phys. Rept. 150, 1 (1987).

[3] K. Rajagopal, M. S. Turner and F. Wilczek, Nucl. Phys. B 358, 447 (1991).

[4] E. J. Chun, H. B. Kim and J. E. Kim, Phys. Rev. Lett. 72, 1956 (1994) arXiv:hep-ph/9305208; L. Covi, J. E. Kim and L. Roszkowski, Phys. Rev. Lett. 82, 4180 (1999) arXiv:hep-ph/9905212.

[5] L. Covi, H. B. Kim, J. E. Kim and L. Roszkowski, JHEP 0105, 033 (2001) arXiv:hep-ph/0101009; A. Brandenburg and F. D. Steffen, JCAP 0408, 008 (2004) arXiv:hep-ph/0405158. 
[6] K. Y. Choi, J. E. Kim, H. M. Lee and O. Seto, arXiv:0801.0491 [hep-ph].

[7] E. J. Chun, H. B. Kim, K. Kohri and D. H. Lyth, arXiv:0801.4108 [hep-ph].

[8] T. Asaka and M. Yamaguchi, Phys. Rev. D 59, 125003 (1999) arXiv:hep-ph/9811451.

[9] M. Kawasaki, K. Nakayama and M. Senami, arXiv:0711.3083 [hep-ph].

[10] M. Endo, K. Hamaguchi and F. Takahashi, Phys. Rev. Lett. 96, 211301 (2006) arXiv:hep-ph/0602061; S. Nakamura and M. Yamaguchi, Phys. Lett. B 638, 389 (2006) arXiv:hep-ph/0602081.

[11] M. Dine, R. Kitano, A. Morisse and Y. Shirman, Phys. Rev. D 73, 123518 (2006) arXiv:hep-ph/0604140.

[12] M. Endo, K. Hamaguchi and F. Takahashi, Phys. Rev. D 74, 023531 (2006) arXiv:hep-ph/0605091.

[13] M. Kawasaki, F. Takahashi and T. T. Yanagida, Phys. Lett. B 638, 8 (2006) arXiv:hep-ph/0603265; Phys. Rev. D 74, 043519 (2006) arXiv:hep-ph/0605297.

[14] M. Endo, F. Takahashi and T. T. Yanagida, Phys. Lett. B 658, 236 (2008) arXiv:hep-ph/0701042.

[15] M. Endo, F. Takahashi and T. T. Yanagida, Phys. Rev. D 76, 083509 (2007) arXiv:0706.0986 [hep-ph]].

[16] D. Seckel and M. S. Turner, Phys. Rev. D 32, 3178 (1985); M. S. Turner and F. Wilczek, Phys. Rev. Lett. 66, 5 (1991).

[17] R. Bean, J. Dunkley and E. Pierpaoli, Phys. Rev. D 74, 063503 (2006) arXiv:astro-ph/0606685]; R. Trotta, Mon. Not. Roy. Astron. Soc. Lett. 375, L26 (2007) arXiv:astro-ph/0608116]; R. Keskitalo, H. Kurki-Suonio, V. Muhonen and J. Valiviita, JCAP 0709, 008 (2007) arXiv:astro-ph/0611917]; M. Kawasaki and T. Sekiguchi, arXiv:0705.2853 [astro-ph].

[18] M. Beltran, J. Garcia-Bellido and J. Lesgourgues, Phys. Rev. D 75, 103507 (2007) arXiv:hep-ph/0606107.

[19] M. Kawasaki and T. Moroi, Prog. Theor. Phys. 93, 879 (1995) arXiv:hep-ph/9403364; Astrophys. J. 452, 506 (1995) arXiv:astro-ph/9412055; E. Holtmann, M. Kawasaki, K. Kohri and T. Moroi, Phys. Rev. D 60, 023506 (1999) arXiv:hep-ph/9805405|; K. Jedamzik, Phys. Rev. Lett. 84, 3248 (2000) arXiv:astro-ph/9909445; M. Kawasaki, K. Kohri and T. Moroi, Phys. Rev. D 63, 103502 (2001) arXiv:hep-ph/0012279]; K. Kohri, Phys. Rev. D 64, 043515 
(2001) arXiv:astro-ph/0103411]; R. H. Cyburt, J. R. Ellis, B. D. Fields and K. A. Olive, Phys. Rev. D 67, 103521 (2003) arXiv:astro-ph/0211258]; K. Jedamzik, Phys. Rev. D 74, 103509 (2006) arXiv:hep-ph/0604251.

[20] M. Kawasaki, K. Kohri and T. Moroi, Phys. Lett. B 625, 7 (2005) arXiv:astro-ph/0402490]; Phys. Rev. D 71, 083502 (2005) [arXiv:astro-ph/0408426].

[21] T. Moroi, H. Murayama and M. Yamaguchi, Phys. Lett. B 303, 289 (1993).

[22] M. Bolz, A. Brandenburg and W. Buchmuller, Nucl. Phys. B 606, 518 (2001) |arXiv:hep-ph/0012052; J. Pradler and F. D. Steffen, Phys. Rev. D 75, 023509 (2007) [arXiv:hep-ph/0608344]; Phys. Lett. B 648, 224 (2007) arXiv:hep-ph/0612291]; V. S. Rychkov and A. Strumia, Phys. Rev. D 75, 075011 (2007) arXiv:hep-ph/0701104.

[23] M. Endo, M. Kawasaki, F. Takahashi and T. T. Yanagida, Phys. Lett. B 642, 518 (2006) arXiv:hep-ph/0607170].

[24] E. J. Chun and A. Lukas, Phys. Lett. B 357, 43 (1995) arXiv:hep-ph/9503233.

[25] E. W. Kolb and M. S. Turner, The Early Universe, (Addison-Wesley, Reading, MA, 1990).

[26] P. J. Steinhardt and M. S. Turner, Phys. Lett. B 129, 51 (1983); G. Lazarides, C. Panagiotakopoulos and Q. Shafi, Phys. Lett. B 192, 323 (1987); G. Lazarides, R. K. Schaefer, D. Seckel and Q. Shafi, Nucl. Phys. B 346, 193 (1990); M. Kawasaki, T. Moroi and T. Yanagida, Phys. Lett. B 383, 313 (1996) arXiv:hep-ph/9510461].

[27] G. G. Raffelt, Phys. Rept. 198, 1 (1990).

[28] K. Kumekawa, T. Moroi and T. Yanagida, Prog. Theor. Phys. 92, 437 (1994) arXiv:hep-ph/9405337; K. I. Izawa and T. Yanagida, Phys. Lett. B 393, 331 (1997) arXiv:hep-ph/9608359; M. Ibe, K. I. Izawa, Y. Shinbara and T. T. Yanagida, Phys. Lett. B 637, 21 (2006) arXiv:hep-ph/0602192.

[29] T. Asaka, K. Hamaguchi, M. Kawasaki and T. Yanagida, Phys. Rev. D 61, 083512 (2000) arXiv:hep-ph/9907559]; V. N. Senoguz and Q. Shafi, Phys. Lett. B 596, 8 (2004) arXiv:hep-ph/0403294;

[30] G. R. Dvali, Q. Shafi and R. K. Schaefer, Phys. Rev. Lett. 73, 1886 (1994) arXiv:hep-ph/9406319; A. D. Linde and A. Riotto, Phys. Rev. D 56, 1841 (1997) arXiv:hep-ph/9703209.

[31] M. Bastero-Gil, S. F. King and Q. Shafi, Phys. Lett. B 651, 345 (2007) arXiv:hep-ph/0604198; M. ur Rehman, V. N. Senoguz and Q. Shafi, Phys. Rev. D 75, 043522 
(2007) arXiv:hep-ph/0612023.

[32] G. Lazarides and C. Panagiotakopoulos, Phys. Rev. D 52, 559 (1995) arXiv:hep-ph/9506325].

[33] M. Kawasaki, M. Yamaguchi and T. Yanagida, Phys. Rev. Lett. 85, 3572 (2000) arXiv:hep-ph/0004243.

[34] A. D. Linde, Phys. Lett. B 259, 38 (1991).

[35] J. E. Kim, Phys. Rev. Lett. 67, 3465 (1991); D. H. Lyth, Phys. Rev. D 48, 4523 (1993) arXiv:hep-ph/9306293; M. Hashimoto, K. I. Izawa, M. Yamaguchi and T. Yanagida, Phys. Lett. B 437, 44 (1998) arXiv:hep-ph/9803263]; T. Banks, M. Dine and M. Graesser, Phys. Rev. D 68, 075011 (2003) arXiv:hep-ph/0210256.

[36] A. R. Zhitnitsky, Sov. J. Nucl. Phys. 31 (1980) 260; M. Dine, W. Fischler and M. Srednicki, Phys. Lett. B 104, 199 (1981).

[37] K. Kohri, T. Moroi and A. Yotsuyanagi, Phys. Rev. D 73, 123511 (2006) arXiv:hep-ph/0507245.

[38] M. Endo and F. Takahashi, Phys. Rev. D 74, 063502 (2006) arXiv:hep-ph/0606075].

[39] T. Moroi and L. Randall, Nucl. Phys. B 570, 455 (2000) arXiv:hep-ph/9906527; M. Fujii and K. Hamaguchi, Phys. Lett. B 525, 143 (2002) arXiv:hep-ph/0110072]; Phys. Rev. D 66, 083501 (2002) [arXiv:hep-ph/0205044].

[40] M. Nagai and K. Nakayama, Phys. Rev. D 76, 123501 (2007) [arXiv:0709.3918 [hep-ph]].

[41] I. Affleck and M. Dine, Nucl. Phys. B 249, 361 (1985); M. Dine, L. Randall and S. D. Thomas, Nucl. Phys. B 458, 291 (1996) arXiv:hep-ph/9507453.

[42] M. Kawasaki and K. Nakayama, Phys. Rev. D 76, 043502 (2007) [arXiv:0705.0079 [hep-ph]].

[43] A. Kusenko, Phys. Lett. B 405, 108 (1997) arXiv:hep-ph/9704273]; Phys. Lett. B 404, 285 (1997) arXiv:hep-th/9704073; S. Kasuya and M. Kawasaki, Phys. Rev. D 61, 041301 (2000) arXiv:hep-ph/9909509]; Phys. Rev. D 62, 023512 (2000) arXiv:hep-ph/0002285.

[44] S. Kasuya, M. Kawasaki and F. Takahashi, in preparation.

[45] K. Yamamoto, Phys. Lett. B 168, 341 (1986); G. Lazarides, C. Panagiotakopoulos and Q. Shafi, Phys. Rev. Lett. 56, 557 (1986).

[46] D. H. Lyth and E. D. Stewart, Phys. Rev. Lett. 75, 201 (1995) arXiv:hep-ph/9502417; Phys. Rev. D 53, 1784 (1996) arXiv:hep-ph/9510204.

[47] K. Choi, E. J. Chun and J. E. Kim, Phys. Lett. B 403, 209 (1997) arXiv:hep-ph/9608222 ; E. J. Chun, D. Comelli and D. H. Lyth, Phys. Rev. D 62, 095013 (2000) 
arXiv:hep-ph/0008133; E. J. Chun, H. B. Kim and D. H. Lyth, Phys. Rev. D 62, 125001 (2000) arXiv:hep-ph/0008139. 